\title{
Rapid Growth of a Facet Joint Cyst Mimicking an Aggressive Tumor in the Lumbar Spine
}

\author{
James M. W. Robins ${ }^{1} \quad$ Senthil K. Selvanathan ${ }^{1}$ \\ ${ }^{1}$ Department of Neurosurgery, Leeds General Infirmary, Leeds, \\ United Kingdom \\ 2Deparment of Pathology, St James' University Hospital, Leeds, \\ United Kingdom
}

\author{
Azzam Ismail ${ }^{2} \quad$ Chris Derham ${ }^{1} \quad$ Deb Pal ${ }^{1}$
}

J Neurosci Rural Pract 2020;11:646-650

\begin{abstract}
Keywords

- facet joint cyst

- tumor

- rapid growth
\end{abstract}

\begin{abstract}
Address for correspondence James M. W. Robins, MRCS, Department of Neurosurgery, Leeds General Infirmary, G Floor, Jubilee Building, Leeds LS1 3EX, United Kingdom (e-mail: jamesrobins@nhs.net).
\end{abstract}

\section{Introduction}

This case is presented as it demonstrates highly unusual behavior of an ordinarily benign and slow-growing lesion namely a facet joint cyst. We present a fast-growing cyst causing neural compression that was successfully treated with lesion excision and decompression of the affected nerve root. Although facet joint cysts are rare, they are clinically important as they can lead to neurological deficits and patient discomfort if left untreated.

The clear message to be presented is that facet joints cysts can grow unusually rapidly, mimicking an aggressive form of primary or secondary spinal tumor; however, timely surgical excision and nerve root decompression lead to excellent patient outcome.

\section{Case Report}

A 67-year-old female patient with a history of fully treated bowel carcinoma presented to the outpatient department with a short history of pain and numbness in the right buttock and down the posterior aspect of the thigh.

Examination demonstrated normal power in all myotomes and normal objective sensation in dermatomes of the lower limbs. Reflexes were also normal bilaterally.

Magnetic resonance imaging (MRI) demonstrated rightsided $\mathrm{L} 4 / 5$ foraminal narrowing and the patient was treated with an L5 nerve root block ( - Fig. 1A). The patient reported no improvement and MRI was repeated 5 months after the original scan. This demonstrated a growth with mixed signal intensity on T1- and T2-weighted imaging, compressing the $\mathrm{S} 1$ nerve root. There was also scalloping of the S1 body, a feature associated with tumor pathology. Diagnosis was felt to be a nerve sheath tumor such as a Schwannoma behaving very atypically. On retrospective review of previous imaging, this lesion was present on the initial MRI; however, it had dramatically increased in size over the past 5 months (-Fig. 1B-E).

The patient was managed conservatively for a further 9 months, as the patient did not want surgical 


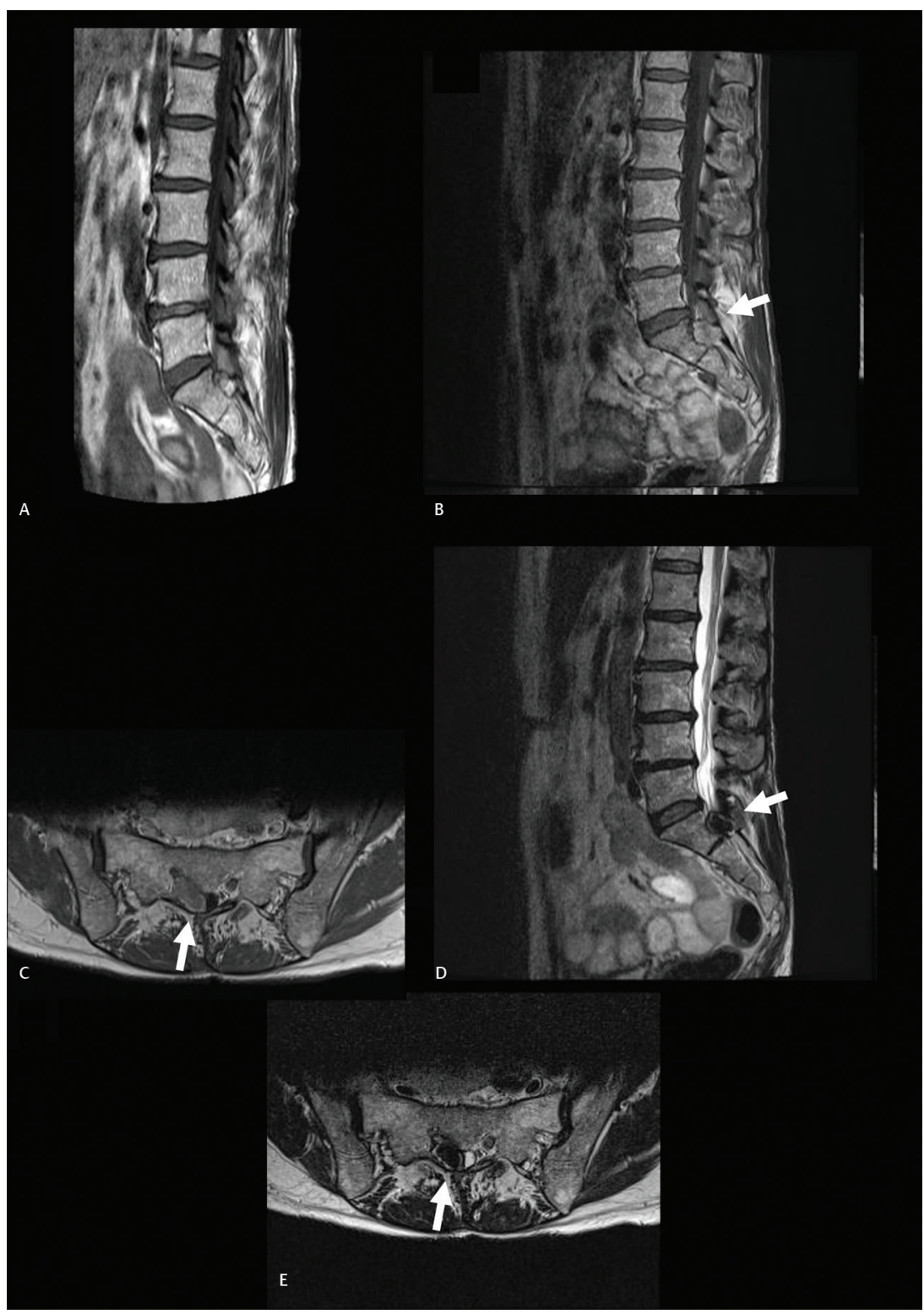

Fig. 1 T1 sagittal magnetic resonance imaging (A) at presentation (no other sequences collected by local hospital). Sagittal and axial T1-weighted image with gadolinium (B, C) and T2 sequences (D, E) at 5 months demonstrating increase in size of lesion (arrow).

intervention; however, ongoing symptoms led to repeat MRI and multidisciplinary team discussion ( - Fig. 2). In a view of previous history of bowel carcinoma, a computed tomography (CT) chest, abdomen, and pelvis was performed that demonstrated no recurrent primary lesion or metastases.

Imaging appearances of an extradural mass associated with the S1 nerve root and scalloping of S1 body were highly suspicious for a nerve sheath tumor and in particular a Schwannoma. However, the rapid growth was suggestive of an aggressive process such as a metastatic lesion. A facet joint cyst was also possibly an explanation; however, the rapid growth and location on imaging did not support this diagnosis.

The worsening of symptoms combined with rapid-interval growth over 9 months on serial imaging led to the patient being listed for urgent open biopsy of presumed nerve sheath tumor and decompression.
At surgery, an L5 and S1 laminectomy was performed ( - Fig. 3A). The lesion was encountered and appeared to be large, cystic, and with collagenous contents ( - Fig. $\mathbf{3 B}$ ). The cyst was gently dissected off the thecal sac and tracked into the S1 foramen. A small amount of lesion was left adherent to thecal sac due to being very adherent and thus posing a high risk of cerebrospinal fluid leak if removed. Good S1 nerve root decompression was achieved. Frozen section biopsy demonstrated collagen and fibrotic tissue. Formal histology was of chronic hemorrhagic simple cyst with no evidence of tumor or inflammation ( - Fig. 3C, D).

Postoperatively, the patient had no neurological deficit and was pain-free. The patient was discharged home the following day after surgery. MRI spine performed at 2 months postoperatively demonstrated good decompression of the S1 nerve root with some residual noncompressive 

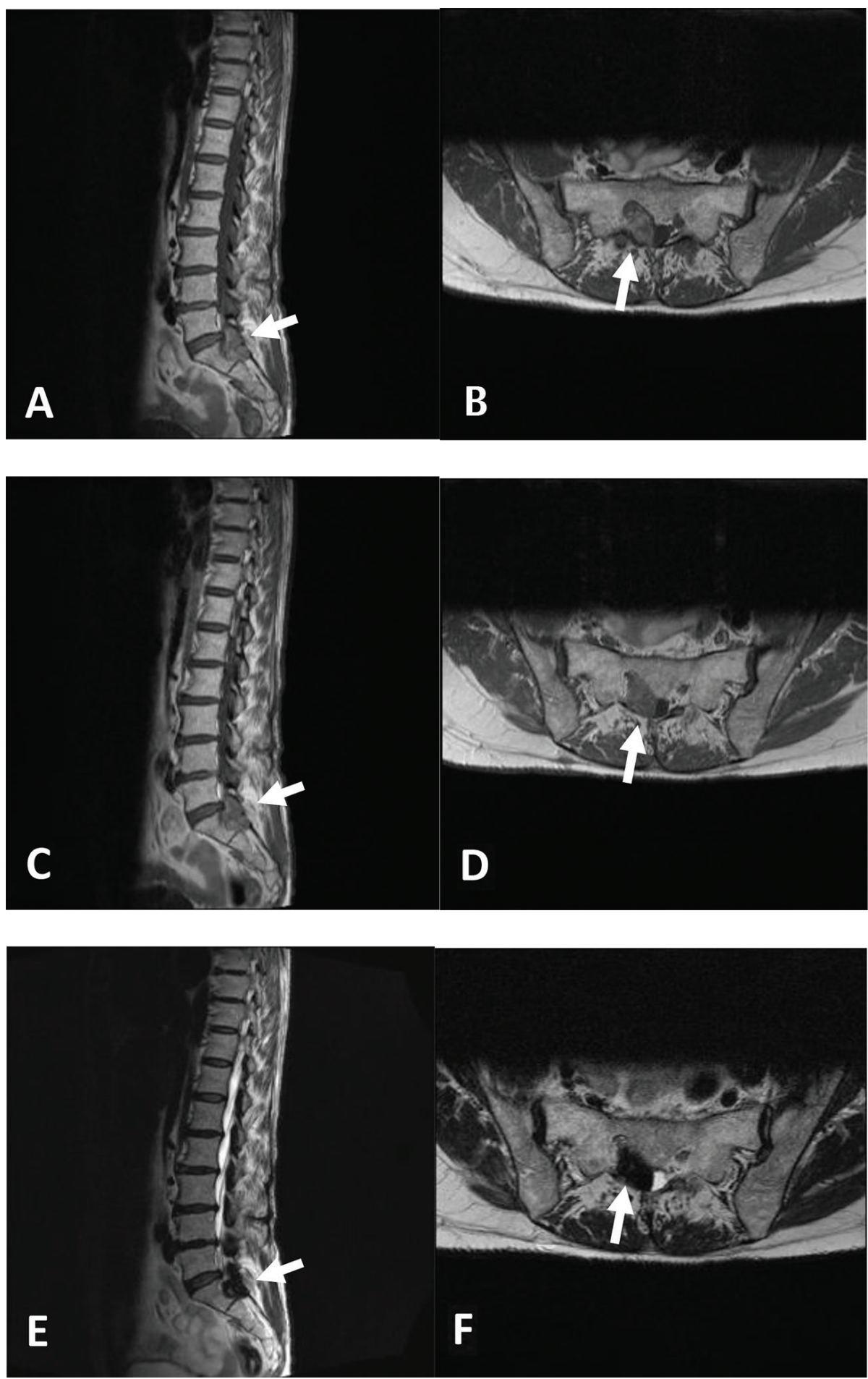

Fig. 2 T1 pre- (A, B) and post-gadolinium (C, D) and T2 sequences (E, F) at 14 months demonstrating rapid interval growth of facet joint cyst (arrow) with scalloping of S1.

cystic material. Clinic follow-up at 3 months postoperatively revealed resolution of symptoms.

\section{Discussion}

Facet joint cysts are rare with prevalence between 0.6 and 7.3\%. ${ }^{1}$ They mostly occur in the lumbar region and less commonly cervical or thoracic and usually present in the sixth decade. ${ }^{2}$ There is debate in the literature as to whether females are more affected than males. ${ }^{1,2}$

Facet joint cysts may be asymptomatic and incidental; however, if symptomatic they present most commonly with lower back pain followed by radiculopathy that may be unilateral or bilateral in 57 to $100 \%$ of patients. ${ }^{3}$ Location of radiculopathy is naturally related to the site of the cyst and its relationship to surrounding neural structures. Facet cysts 


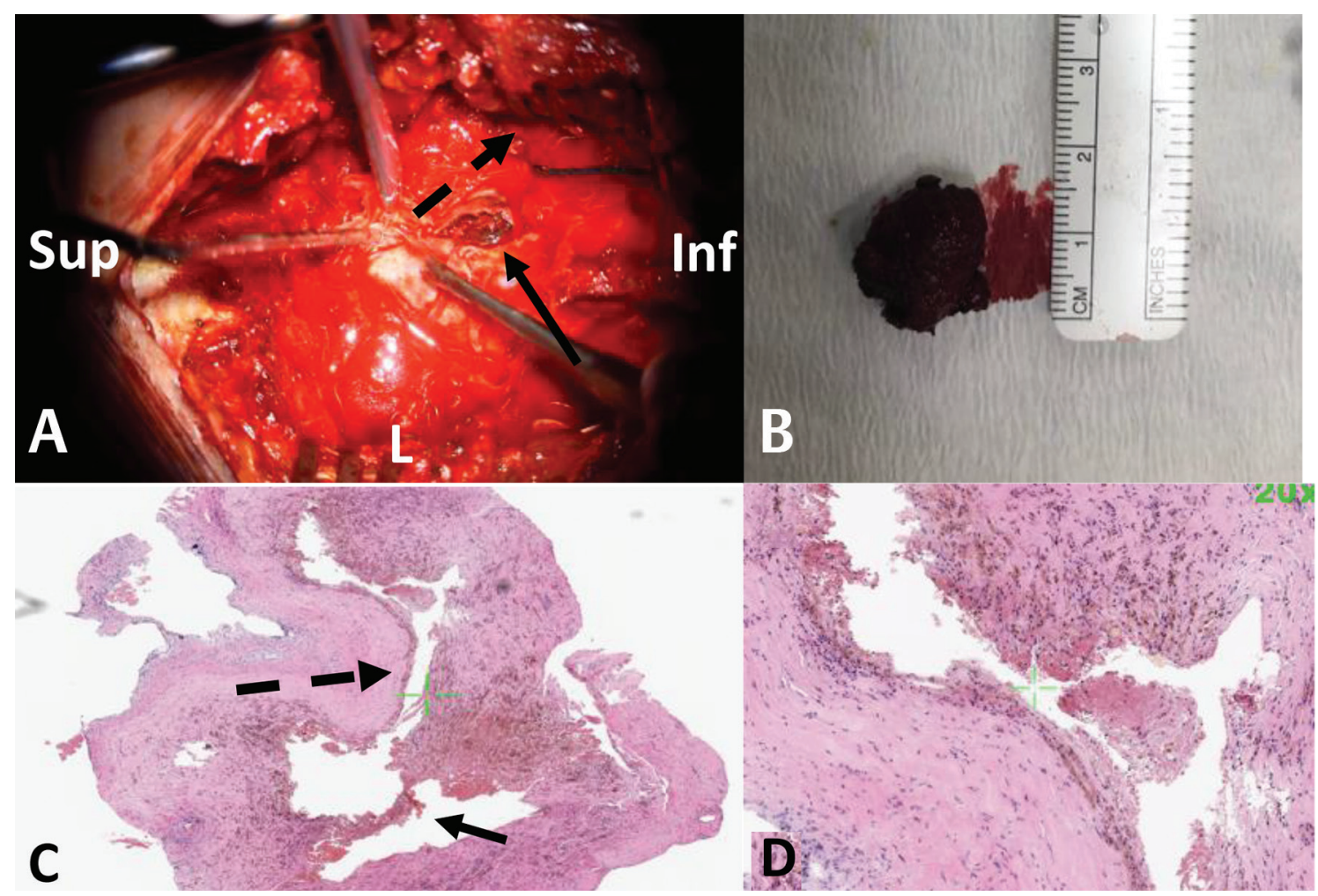

Fig. 3 Intraoperative photograph (A) demonstrating opened cyst in situ (arrow), direction of S1 nerve root (dashed arrow), appearance after excision demonstrating collagenous contents (B), and histology (C, D) demonstrating simple cyst containing fragments of chronic hematoma (arrow) and lined by simple cuboidal epithelium (dashed arrow).

may also present with neurogenic claudication in 10 to $44 \%$ and less commonly with sensory (10-43\%), motor (20-27\%), or reflex (57\%) neurological deficit. ${ }^{2,3}$ Rarely, compression can be extensive enough to cause cauda equina syndrome; however, this incidence is reported as between 1 and 13\%, and even less commonly reported is lateral recess stenosis. ${ }^{3}$

MRI is the investigation of choice and classically demonstrates an extradural, well-circumscribed, smooth lesion adjacent to the facet joint. A high protein content may demonstrate a high signal intensity on $\mathrm{T} 1$ and $\mathrm{T} 2$ sequences; however, this can vary depending on cyst content namely gas, calcification, blood, or inflammatory tissue. ${ }^{4}$ They are not reported in the literature as causing bone scalloping; indeed, this is a key feature normally associated with tumor pathology. Indeed, bone scalloping has not previously been reported in the literature with facet joint cysts. ${ }^{5}$

Facet cysts are typically found in the L4-5 region and this correlates with the site of maximum mobility in the lumbar spine and also site of maximum degenerative disease including spondylosis and spondylolisthesis that may be present in up to $60 \%$ of patients. ${ }^{3}$ Coexisting facet joint arthropathy has been noted in $90 \%$ of patients. ${ }^{6}$ Next most affected levels follow degenerative locations, L5-S1 then L3-4; however, multiple levels can also be affected. ${ }^{2}$

Pathologically, facet joint cysts are composed of cuboid or pseudocolumnar epithelium and contain clear or straw-colored fluid. They can be classified as either synovial or ganglionic histologically; however, it is felt that the these different appearances may represent different parts of the cystic formation and degeneration cycle and regardless management is the same for both. ${ }^{7}$ Cysts can be found to have hemorrhaged and this can lead to acute neural compression with an incidence of $9.3 \%$ reported. $^{2}$

Management options for asymptomatic cysts are conservative; however, for symptomatic cysts, analgesia and physiotherapy have a role. There are reports in the literature of cysts resolving spontaneously. ${ }^{8}$ For persistent cysts, needle aspiration or puncture under CT guidance is described; however, cysts typically recur after 6 to 12 months probably due to residual synovial tissue. ${ }^{9}$ The use of intraarticular steroids is also described in the literature. ${ }^{9}$

Despite these nonsurgical options, surgical excision and decompression remain the advised management in the symptomatic patient especially with a neurological deficit. Technique involves partial or total neural decompression and excision of the lesion. ${ }^{2}$ Minimally invasive techniques are also described including a hemilaminectomy. ${ }^{10}$ Some advocate concomitant fixation if there is evidence of instability; however, there is no difference compared with nonfixed patients in terms of outcome.

In this case, the presentation of this symptomatic facet joint cyst is typical with a unilateral painful radiculopathy. However, it is highly unusual to see the rapid progression of symptoms secondary to rapid growth of this lesion. These lesions are known to be very slow growing and this leads to the possibility of an atypical nerve sheath tumor or possibly a metastatic lesion, in this case secondary to colon carcinoma. Preoperative CT evaluation demonstrated no recurrence of 
this primary carcinoma for this patient and in view of the abnormal behavior on MRI, open biopsy and decompression were performed. Intraoperative appearances and subsequent formal histology demonstrated a simple chronic hemorrhagic cyst. There are no reports in the literature of this type for lesion growing so rapidly nor with scalloping of bone on MRI. The urgent growth of this lesion guided the urgent subsequent excision and decompression that led to a good outcome for this patient.

\section{Conclusion}

Facet joint cysts, although uncommon, are an important cause of lumbosacral neural compression and typically present with a painful radiculopathy. The investigation of choice is MRI. Facet joint cysts may behave unpredictably demonstrating rapid growth mimicking other lesions such as tumors and cause rapid progression of symptoms. Timely intervention for a rapid growing facet joint cyst led to a good patient outcome in this case.

\section{Conflict of Interest}

None declared.

\section{References}

1 Eyster EF, Scott WR. Lumbar synovial cysts: report of eleven cases. Neurosurgery 1989;24(1):112-115

2 Lyons MK, Atkinson JL, Wharen RE, Deen HG, Zimmerman RS, Lemens SM. Surgical evaluation and management of lumbar synovial cysts: the Mayo Clinic experience. J Neurosurg 2000;93(1(Suppl):53-57
3 Trummer M, Flaschka G, Tillich M, Homann CN, Unger F, Eustacchio S. Diagnosis and surgical management of intraspinal synovial cysts: report of 19 cases. J Neurol Neurosurg Psychiatry 2001;70(1):74-77

4 Knox AM, Fon GT. The appearances of lumbar intraspinal synovial cysts. Clin Radiol 1991;44(6):397-401

5 Kang SH, Lee SM, Ha DH, Lee HJ. Extensive spinal extradural ganglioneuroma of the lumbar spine: mimicking lymphoma. Eur Spine J 2018;27(Suppl 3) :520-525

6 Banning CS, Thorell WE, Leibrock LG. Patient outcome after resection of lumbar juxta facet cysts. Spine 2001;26(8): 969-972

7 Sabo RA, Tracy PT, Weinger JM. A series of 60 juxta facet cysts: clinical presentation, the role of spinal instability, and treatment. J Neurosurg 1996;85(4):560-565

8 Ewald C, Kalff R. Resolution of a synovial cyst of the lumbar spine without surgical therapy - a case report. Zentralbl Neurochir 2005;66(3):147-151

9 Abrahams JJ, Wood GW, Eames FA, Hicks RW. CT-guided needle aspiration biopsy of an intraspinal synovial cyst (ganglion): case report and review of the literature. AJNR Am J Neuroradiol. 9(2):398-400

10 Oertel MF, Ryang Y, Ince A, Gilsbach JM, Rohde V. Microsurgical therapy of symptomatic lumbar juxta facet cysts. Minim Invasive Neurosurg 2003;46(6):349-353 